\title{
Effect of anti-osteoporotic agents on the prevention of bone loss in unloaded bone
}

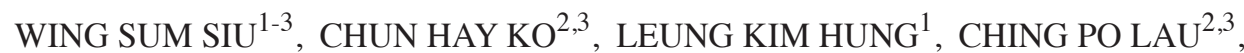 \\ CLARA BIK SAN LAU ${ }^{2,3}$, KWOK PUI FUNG ${ }^{2,3}$ and PING CHUNG LEUNG ${ }^{1-3}$
}

\author{
${ }^{1}$ Department of Orthopaedics and Traumatology; ${ }^{2}$ Institute of Chinese Medicine; ${ }^{3}$ State Key Laboratory of Phytochemistry \\ and Plant Resources in West China, The Chinese University of Hong Kong, Shatin, NT, Hong Kong SAR, P.R. China
}

Received February 28, 2013; Accepted June 20, 2013

DOI: $10.3892 / \mathrm{mmr} .2013 .1647$

\begin{abstract}
Pharmaceutical countermeasures to treat disuse osteoporosis are rarely studied. Pharmaceutical studies for the treatment and prevention of osteoporosis depend on the ovariectomized rat model, which is a suitable model for the disease in women. Disuse osteoporosis affects men and women, but there is lack of awareness and relevant pharmaceutical studies for this condition. The objectives of this study were to verify the validity of an unusual tail-suspension rat model in the induction of disuse osteoporosis and subsequent pharmaceutical treatments. This model was created by unloading the hind limbs of the rats in order to create a state of weightlessness in their hindlimb bones. Validation of the model was performed with nonsuspended rats. This study included five groups of suspended rats fed with different agents, such as distilled water (control), high-, medium- and low-dose raloxifene and a bisphosphonate (alendronate). The experiment lasted for 28 days. Comparisons were made between the suspended control and treatment groups. Ovariectomized and sham-operated rats were also included as a reference for bone changes during osteoporosis. Changes in bone mineral density (BMD) at the distal femur and proximal tibia, microarchitecture at the distal femur and biomechanical strength at the diaphyseal femur were studied. Reduction of BMD and deterioration of trabeculae were similar between the suspended control and ovariectomized rats. Loss of BMD induced by tail suspension was reduced most effectively by medium-dose raloxifene. Deterioration of trabecular microarchitecture was also prevented by raloxifene. The tail-suspension rat model is suitable for the study of disuse osteoporosis under the effects of various therapeutic agents. The preventive effects of raloxifene against bone loss under disuse conditions have been demonstrated using this model.
\end{abstract}

Correspondence to: Professor Ping Chung Leung, 5/F The CUHK Hong Kong Jockey Club School of Public Health Building, Prince of Wales Hospital, Shatin, NT, Hong Kong SAR, P.R. China

E-mail: pingcleung@cuhk.edu.hk

Key words: osteoporosis, raloxifene, tail-suspension, bone mineral density, micro-architecture

\section{Introduction}

Disuse osteoporosis is defined as localized or generalized bone loss induced by a reduction of mechanical loading on the skeleton (1). Since physical activity is one of the important factors required to maintain an adequate bone remodeling rate and bone mass $(2,3)$, prolonged removal of mechanical stimuli on bone cells may lead to osteoporosis $(4,5)$.

Microgravity is one of the main causes of disuse osteoporosis. The development of disuse osteoporosis among astronauts during spaceflight is well known $(6,7)$. One study showed that astronauts experienced $1-2 \%$ loss of bone mass each month in the microgravity environment and this resulted in osteopenia (8). Another study also reported 6.8 and $7.8 \%$ of bone mineral density (BMD) loss at the trochanter and femoral neck regions, respectively, in cosmonauts and astronauts following flights (9).

Another possible factor related to osteoporosis is low body weight. Mechanical loading on the skeleton is reduced when body weight decreases. Low body weight has been found to be associated with a high prevalence of osteoporosis $(10,11)$. A recent publication reported that men who were underweight or normal weight have a higher chance of osteoporosis and a higher fracture risk than those who were overweight or obese (12).

The tail-suspension (TS) rat is a new animal model for studying the biological mechanisms, treatment or prevention of disuse osteoporosis. This model has been used in studies relating to microgravity, since it mimics certain aspects of exposure to microgravity by inducing a state of weightlessness on the hindquarters of the animal, thereby producing a cephalic fluid shift (13). Effects of disuse on the unloaded hindlimb bones have also been well observed $(14,15)$. Therefore, this animal model may be utilized to study bone changes associated with disuse, weightlessness or low body weight. This new model provides a good alternative for the study of osteoporosis, which uses ovariectomized female rats as the standard in vivo model.

Estrogen is important in the adaptive response of bones to load. It may lower the threshold of bone sensitivity to mechanical loads (16) and was suggested to have an amplifying effect on the osteogenic response to strain (17). Estrogen receptor $\alpha$ has also been shown to be involved in the response of bone 
cells to mechanical strain (18). To avoid the adverse effects of estrogen, synthetic pharmaceutical agents resembling estrogen, selected estrogen receptor modulators (SERMs), were created to prevent bone loss due to disuse. Raloxifene is one of the SERMs commonly used to tackle postmenopausal osteoporosis $(19,20)$.

In this study, we investigated whether raloxifene exhibited an osteoprotective effect on osteoporotic bone induced by the state of weightlessness in the tail-suspension rat model. At the same time, bone strength, which is dependent on both the material and micro-architectural properties of bone, was studied.

\section{Materials and methods}

Animals and grouping. In total, 46 male and 18 female 3-month-old Sprague-Dawley rats (weighing 446.0 $\pm 38.4 \mathrm{~g}$ and $225.2 \pm 15.4$ g, respectively) were obtained from the Laboratory of Animal Services Centre of the Chinese University of Hong Kong. The rats were housed in a temperature- $\left(25^{\circ} \mathrm{C}\right)$ and light-controlled (12-h light/dark cycle) environment. After seven days of acclimatization, the male rats were divided into six groups: tail-suspension control (TS) group, non-tail suspension control (Non-TS) group, tail-suspension with high-, medium- or low-dose raloxifene hydrochloride (Evista, Eli Lilly, Indianapolis, IN, USA) (TS-RH, TS-RM and TS-RL, respectively) groups or the alendronate (Fosamax, Merck Sharp \& Dohme, Whitehouse Station, NJ, USA) (TS-A) group as the positive control. The female rats were divided into two groups: ovariectomy (OVX) and sham control (Sham). At least six rats were included in each group.

In the Non-TS group, rats were allowed to move freely (without hindlimb unloading). In the TS, TS-RH, TS-RM and TS-RL groups, the tails of the rats were suspended in order that their hind limbs were unloaded. Using a gastric tube, rats in the TS-RH group received raloxifene at a dose of $2.5 \mathrm{mg} / \mathrm{kg} /$ day orally while those in the TS-RM and TS-RL groups received raloxifene at a dose of 1.25 and $0.625 \mathrm{mg} / \mathrm{kg}$ / day, respectively. Rats in the TS-A group received alendronate at a dose of $1.0 \mathrm{mg} / \mathrm{kg} / \mathrm{day}$. The dose of the TS-RM and TS-A groups was designed based on the human clinical dose, which is $\sim 1.0$ and $0.17 \mathrm{mg} / \mathrm{kg} / \mathrm{day}$, respectively. The drugs were dissolved in distilled water. Rats in the TS and Non-TS groups received distilled water vehicle $(2.0 \mathrm{ml} /$ day $)$. Rats in the OVX group were ovariectomized bilaterally, while those in the Sham group underwent sham surgery. Sham and OVX groups were non-tail suspended.

The animals were allowed access to standard rodent chow and water ad libitum throughout the study. At the end of the study, rats were euthanized by an overdose of ketamine and xylazine cocktail intravenously. Body weights of the animals were recorded at the beginning and end of the study. Animal ethics approval was obtained from the Animal Experimental Ethics Committee of the Chinese University of Hong Kong (ref no. 07/052/MIS) for this study.

Tail-suspension model. The protocol of the tail-suspension was modified from that of Morey-Holton and Globus (21). The tail of each rat was suspended by applying an adhesive tape on its lateral surfaces and then secured by three surgical tapes applied circularly. The loop of the adhesive tape at the tip of the tail passed through a metallic hollow column, which was then connected to a free rotating hook. The hook was hung on an overhead bar. As a result, the rat was maintained in an $\sim 35^{\circ}$ head-down tilt with its hind limbs unloaded, while its forelimbs could be used for locomotion. The overall suspension period was 28 days. This new model of hindlimb bone osteoporosis was to be compared with the conventional ovariectomy model. Ovariectomy was performed under general anesthesia in the usual manner. A sham-operation group was created as a control.

\section{Study of bone structure}

Peripheral quantitative computed tomography ( $p Q C T)$. Changes in BMD at the distal femoral metaphysis and proximal tibial metaphysis of the rat were studied using pQCT (XCT2000, Stratec Medizintechnik GmbH, Pforzheim, Germany). Quality assurance of measurements was confirmed by using the hydroxyapatite cone and standard phantoms prior to the scanning of the rats each time. The rat was fixed on a custom-made translucent plastic holder to ensure repeatable positioning. The right distal femur and proximal tibia were scanned at a voxel resolution of $0.2 \mathrm{~mm}$. The analytical parameters for trabecular BMD were set as threshold $280 \mathrm{mg} /$ $\mathrm{cm}^{3}$, contour mode 1 and peel mode 20 . The parameters for cortical BMD were set as threshold $551 \mathrm{mg} / \mathrm{cm}^{3}$ and peel mode 2 . The trabecular bone region was defended by setting an inner area to $35 \%$ of the total cross-sectional area.

Micro-computed tomography (micro-CT). The micro-architecture of the left distal femur was analyzed using a micro-CT (MicroCT 40, Scanco Medical, Bassersdorf, Switzerland). The scanning was conducted at $55 \mathrm{kVp}$ and $144 \mu \mathrm{A}$ with a resolution of $16 \mu \mathrm{m}$ per voxel. Segmentation parameters were: Sigma, 0.5; support, 1.0; and threshold, 245. The micro-architectural parameters, including bone volume density (BV/TV), connectivity density (Conn.D.), structure model index (SMI), trabecular number (Tb.N), trabecular thickness (Tb.Th) and trabecular plate separation (Tb.Sp), were obtained.

Biomechanical test. The right femur of the rat was harvested for a three-point bending test using a Hounsfield material testing machine (KM25, Redhill, United Kingdom) with a $250 \mathrm{~N}$ load-cell. The span of the lower supports was $20 \mathrm{~mm}$. The mid-shaft of the bone was loaded at a constant speed of $5 \mathrm{~mm} / \mathrm{min}$ in a medial-lateral approach until failure. Loads at yield, maximum (ultimate) and break (failure) were recorded for analysis. Young's modulus (stiffness) was calculated as the steepest slope of the elastic region of the strength-displacement curve of the test.

Statistical analysis. The differences between the TS and Non-TS groups, and the OVX and Sham groups were analyzed by the Student's t-test. Percentage differences of BMD between different time intervals in each group and comparisons of BMD, micro-architectural and biomechanical parameters between drug treatment groups and the TS group were tested using one-way ANOVA, followed by the post-hoc Dunnett's test. There was no direct statistical comparison between the TS and OVX groups on micro-architectural and biomechanical parameters, since the two groups consisted of different 
Table I. Means of percentage difference from baseline \pm standard error of mean of the bone mineral density at different regions of interest (ROI) in rats assessed using peripheral quantitative computed tomography.

\begin{tabular}{|c|c|c|c|c|c|c|}
\hline Body part & ROI & Days & Sham & OVX & Non-TS & TS \\
\hline \multirow[t]{6}{*}{ Distal femur } & \multirow[t]{2}{*}{ Total } & 14 & $-0.72 \pm 0.55$ & $-10.04 \pm 1.04^{\mathrm{b}, \mathrm{d}}$ & $0.67 \pm 1.07$ & $-8.08 \pm 0.85^{\mathrm{a}, \mathrm{f}}$ \\
\hline & & 28 & $1.17 \pm 0.84$ & $-10.90 \pm 1.50^{\mathrm{b}, \mathrm{d}}$ & $3.02 \pm 1.46$ & $-13.19 \pm 1.46^{\mathrm{b}, \mathrm{f}}$ \\
\hline & \multirow[t]{2}{*}{ Trabecular } & 14 & $-0.35 \pm 0.71$ & $-13.32 \pm 1.51^{\mathrm{b}, \mathrm{d}}$ & $0.16 \pm 1.66$ & $-15.74 \pm 1.46^{\mathrm{b}, \mathrm{f}}$ \\
\hline & & 28 & $1.16 \pm 1.48$ & $-15.38 \pm 2.38^{\mathrm{b}, \mathrm{d}}$ & $2.47 \pm 2.22$ & $-24.46 \pm 1.84^{b, f}$ \\
\hline & \multirow[t]{2}{*}{ Cortical } & 14 & $-0.51 \pm 0.38$ & $-4.66 \pm 0.45^{b, d}$ & $1.54 \pm 0.47$ & $-0.98 \pm 0.52^{\mathrm{f}}$ \\
\hline & & 28 & $0.92 \pm 0.56$ & $-5.24 \pm 0.78^{\mathrm{b}, \mathrm{d}}$ & $3.30 \pm 0.63$ & $-1.82 \pm 0.51^{\mathrm{f}}$ \\
\hline \multirow[t]{6}{*}{ Proximal tibia } & \multirow[t]{2}{*}{ Total } & 14 & $1.65 \pm 0.80$ & $-9.77 \pm 1.29^{\mathrm{b}, \mathrm{d}}$ & $2.42 \pm 1.72$ & $-11.64 \pm 1.23^{\mathrm{b}, \mathrm{f}}$ \\
\hline & & 28 & $2.87 \pm 1.02$ & $-16.40 \pm 1.54^{\mathrm{b}, \mathrm{d}}$ & $5.08 \pm 2.01$ & $-13.57 \pm 1.59^{b, f}$ \\
\hline & \multirow[t]{2}{*}{ Trabecular } & 14 & $0.49 \pm 2.14$ & $-21.35 \pm 2.11^{\mathrm{b}, \mathrm{d}}$ & $1.39 \pm 3.37$ & $-16.28 \pm 3.57^{a, e}$ \\
\hline & & 28 & $0.45 \pm 1.83$ & $-36.21 \pm 2.35^{\mathrm{b}, \mathrm{d}}$ & $-2.76 \pm 5.38$ & $-26.72 \pm 3.44^{\mathrm{b}, \mathrm{e}}$ \\
\hline & \multirow[t]{2}{*}{ Cortical } & 14 & $1.60 \pm 0.54$ & $-0.45 \pm 0.72^{c}$ & $3.21 \pm 0.69$ & $-3.15 \pm 0.74^{\mathrm{f}}$ \\
\hline & & 28 & $3.24 \pm 0.41$ & $-1.26 \pm 0.86^{\mathrm{d}}$ & $6.07 \pm 0.85$ & $-1.80 \pm 1.40^{\mathrm{f}}$ \\
\hline
\end{tabular}

Sham, sham operation; OVX, ovariectomy; Non-TS, non-tail suspension; TS, tail-suspension. ${ }^{\mathrm{a}} \mathrm{P}<0.05,{ }^{\mathrm{b}} \mathrm{P}<0.01 \mathrm{vs}$. baseline; ${ }^{\mathrm{c}} \mathrm{P}<0.05$, ${ }^{\mathrm{d}} \mathrm{P}<0.01$ vs. Sham; ${ }^{\mathrm{e}}<0.05,{ }^{\mathrm{f}} \mathrm{P}<0.01$ vs. Non-TS.

Table II. Means \pm standard error of mean and percentage difference of mean (\%) of the structural indices of different groups of rats assessed using micro-computed tomography.

\begin{tabular}{|c|c|c|c|c|c|c|}
\hline Structural index & Sham & OVX & $\%$ & Non-TS & $\mathrm{TS}$ & $\%$ \\
\hline BV/TV (1) & $0.32 \pm 0.01$ & $0.26 \pm 0.02^{\mathrm{a}}$ & -18.0 & $0.33 \pm 0.01$ & $0.24 \pm 0.01^{\mathrm{b}}$ & -26.7 \\
\hline Conn.D. $\left(1 / \mathrm{mm}^{3}\right)$ & $87.68 \pm 5.17$ & $80.34 \pm 6.06$ & -8.4 & $57.82 \pm 2.89$ & $62.78 \pm 2.65$ & 8.6 \\
\hline SMI (1) & $0.28 \pm 0.15$ & $0.91 \pm 0.16^{\mathrm{a}}$ & 220.8 & $0.38 \pm 0.08$ & $1.00 \pm 0.13^{\mathrm{b}}$ & 160.3 \\
\hline Tb.N (1/mm) & $4.49 \pm 0.06$ & $3.76 \pm 0.34$ & -16.2 & $3.76 \pm 0.05$ & $3.54 \pm 0.07$ & -5.8 \\
\hline Tb.Th (mm) & $0.08 \pm 0.002$ & $0.08 \pm 0.004$ & -5.9 & $0.10 \pm 0.003$ & $0.08 \pm 0.002^{\mathrm{b}}$ & -17.4 \\
\hline Tb.Sp (mm) & $0.22 \pm 0.004$ & $0.24 \pm 0.01$ & 6.0 & $0.25 \pm 0.005$ & $0.28 \pm 0.01^{\mathrm{b}}$ & 12.0 \\
\hline
\end{tabular}

Sham, sham operation; OVX, ovariectomy; Non-TS, non-tail suspension; TS, tail-suspension; BV/TV, bone volume density; Conn.D., connectivity density; SMI, structure model index; Tb.N, trabecular number; Tb.Th, trabecular thickness; Tb.Sp, trabecular plate separation. ${ }^{a} \mathrm{P}<0.05$ vs. Sham; ${ }^{\text {b }}<0.01$ vs. Non-TS.

genders and had different bone sizes. Statistical analyses were performed by using the Statistical Package of Social Science (SPSS) version 16.0 for Windows and were carried out at the $5 \%$ level of significance $(\mathrm{P}<0.05)$. Data are expressed as the means \pm standard error of mean (SEM).

\section{Results}

Body weight. There was no difference in body weight among the various tail suspension groups. All the animals were observed without any adverse effects throughout the study.

\section{Validation of the tail-suspension rat model}

Changes of BMD. Femoral and tibial BMD was significantly reduced in total and in the trabecular regions in both the OVX and TS groups at day 14 and $28(\mathrm{P}<0.05$ for femoral total and tibial trabecular of the TS group at day $14, \mathrm{P}<0.01$ for others) (Table I). The mean percentage difference of total and trabecular BMD of the two groups was similar at each time point. Moreover, the region with the most BMD loss of the two groups was the trabecular region. For the cortical BMD, there was no significant difference from baseline in both the OVX and TS groups at the proximal tibia. The cross-sectional comparison showed that the mean percentage difference of $\mathrm{BMD}$ at all three regions of the OVX and TS groups was significantly lower than that in the Sham and Non-TS groups, respectively, at days 14 and 28 (Table I).

Differences in micro-architecture. BV/TV was reduced significantly in the OVX (-18.0\%) and TS (-26.7\%) groups when compared with the Sham and Non-TS groups, respectively $(\mathrm{P}<0.05$ for both) (Table II). A significant increase in SMI was also observed when the OVX group was compared with the Sham group, as well as when the TS group was compared with the Non-TS group. Tb.N and Tb.Th were reduced in the OVX and TS groups, although a significant difference was found in Tb.Th of the TS group only. Tb.Sp was found to be increased slightly in the OVX and TS groups, but a significant difference was observed in the TS group only. 

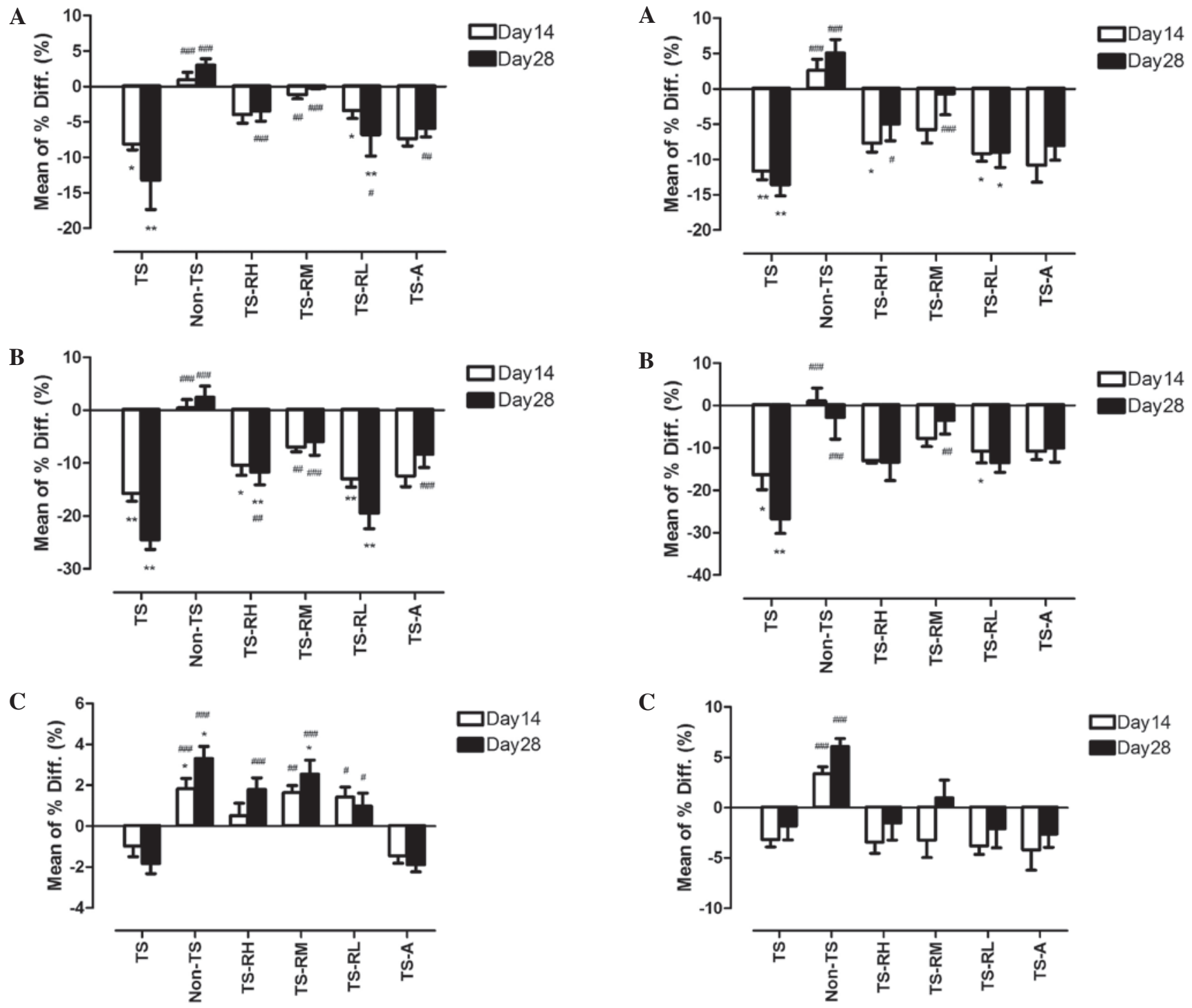

Figure 1. Mean percentage differences of bone mineral density (BMD) in the distal femur between the baseline and days 14 and 28, respectively. The error bar presents the standard error of the mean. (A) Total BMD. (B) Trabecular BMD. (C) Cortical BMD. TS, tail-suspension; Non-TS, non-tail suspension; TS-RH, tail-suspension with high-dose raloxifene; TS-RM, tail-suspension with medium-dose raloxifene; TS-RL, tail-suspension with low-dose raloxifene; TS-A, tail-suspension with alendronate. ${ }^{*} \mathrm{P}<0.05,{ }^{* *} \mathrm{P}<0.01$ (day 14 and 28 vs. baseline of the same group). ${ }^{\#} \mathrm{P}<0.05,{ }^{\# \#} \mathrm{P}<0.01,{ }^{\# \# "} \mathrm{P}<0.001$ (vs. TS group at the same time point).

Effects of raloxifene or alendronate on tail-suspensioninduced disuse osteoporosis

Changes of BMD.

Distal femur. Total BMD was lost significantly only in the TS-RL group at days 14 and $28(\mathrm{P}<0.05$ and $\mathrm{P}<0.01$, respectively) (Fig. 1A). When compared with the TS group horizontally, the mean percentage difference of total BMD was significantly lower in other drug-treated groups at day 28 ( $\mathrm{P}<0.001$ for the TS-RH and TS-RM groups; $\mathrm{P}<0.05$ for the TS-RL group; $\mathrm{P}<0.01$ for the TS-A group).

In the trabecular region, there was no significant reduction of BMD in the TS-RM and TS-A groups throughout the study (Fig. 1B). However, the BMD was reduced significantly in the TS-RH ( $\mathrm{P}<0.05$ and $\mathrm{P}<0.01$ for days 14 and 28 , respectively) and TS-RL ( $\mathrm{P}<0.01$ for both time points) groups compared

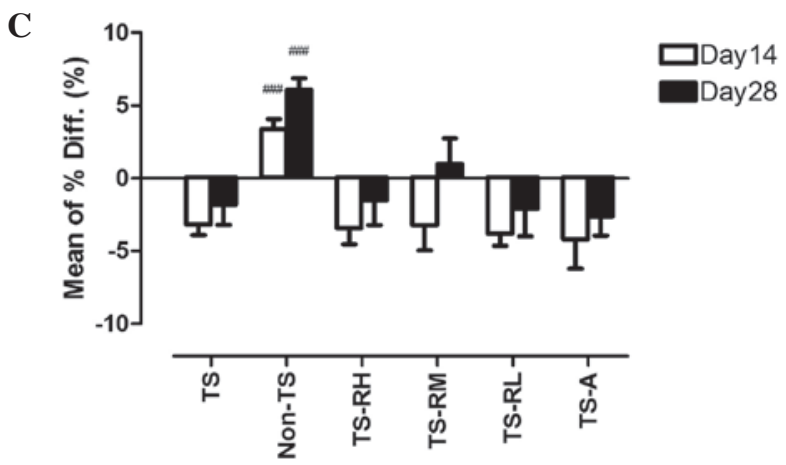

Figure 2. Mean percentage differences of bone mineral density (BMD) in the proximal tibia between the baseline and days 14 and 28, respectively. The error bar presents the standard error of mean. (A) Total BMD. (B) Trabecular BMD. (C) Cortical BMD. TS, tail-suspension; Non-TS, non-tail suspension; TS-RH, tail-suspension with high-dose raloxifene; TS-RM, tail-suspension with medium-dose raloxifene; TS-RL, tail-suspension with low-dose raloxifene; TS-A, tail-suspension with alendronate. ${ }^{*} \mathrm{P}<0.05,{ }^{* *} \mathrm{P}<0.01$, (day 14 and 28 vs. baseline of the same group). ${ }^{\#} \mathrm{P}<0.05,{ }^{\# \#} \mathrm{P}<0.01,{ }^{\# \# "} \mathrm{P}<0.001$ (vs. TS group at the same time point).

with baseline. A cross-sectional comparison with the TS group showed that the mean percentage difference of the TS-RH, TS-RM and TS-A groups was significantly lower than the TS group at day $28(\mathrm{P}<0.01$ for TS-RH group, $\mathrm{P}<0.001$ for both TS-RM and TS-A groups). The same observation was made at day 14 in the TS-RM group $(\mathrm{P}<0.01)$.

The cortical BMD of drug-treated groups was either unchanged or increased slightly when compared with baseline (Fig. 1C). The mean percentage difference of all raloxifene-treated groups, with the exception of the TS-RH group at day 14, was significantly higher than the TS group.

The cross-sectional comparison showed that there was no significant difference in BMD in the three regions when the raloxifene-treated groups were compared with the TS-A group. 
Table III. Means \pm standard error of mean (percentage difference of mean from TS group) of the structural indices of different groups of rats assessed using micro-computed tomography.

\begin{tabular}{lcrcccccr}
\hline & \multicolumn{7}{c}{ Group } \\
\cline { 2 - 9 } Structural index & \multicolumn{2}{c}{ TS-RH } & \multicolumn{2}{c}{ TS-RM } & \multicolumn{2}{c}{ TS-RL } & \multicolumn{2}{c}{ TS-A } \\
\hline BV/TV $(1)$ & $0.30 \pm 0.02^{\mathrm{a}}$ & $(22.7 \%)$ & $0.30 \pm 0.01^{\mathrm{a}}$ & $(24.3 \%)$ & $0.29 \pm 0.01$ & $(18.8 \%)$ & $0.31 \pm 0.01^{\mathrm{b}}$ & $(25.1 \%)$ \\
Conn.D. $\left(1 / \mathrm{mm}^{3}\right)$ & $62.28 \pm 4.29$ & $(-0.8 \%)$ & $78.07 \pm 6.38$ & $(24.4 \%)$ & $70.17 \pm 8.74$ & $(11.8 \%)$ & $54.66 \pm 2.06$ & $(-12.9 \%)$ \\
SMI $(1)$ & $0.74 \pm 0.14$ & $(-26.1 \%)$ & $0.76 \pm 0.13$ & $(-24.5 \%)$ & $0.83 \pm 0.07$ & $(-16.8 \%)$ & $0.39 \pm 0.13^{\mathrm{b}}$ & $(-60.7 \%)$ \\
Tb.N $(1 / \mathrm{mm})$ & $3.73 \pm 0.10$ & $(5.2 \%)$ & $4.17 \pm 0.12^{\mathrm{b}}$ & $(17.7 \%)$ & $3.99 \pm 0.19^{\mathrm{a}}$ & $(12.7 \%)$ & $3.60 \pm 0.07$ & $(1.7 \%)$ \\
Tb.Th (mm) & $0.10 \pm 0.005^{\mathrm{a}}$ & $(13.6 \%)$ & $0.10 \pm 0.001^{\mathrm{b}}$ & $(15.6 \%)$ & $0.09 \pm 0.003$ & $(8.9 \%)$ & $0.09 \pm 0.001^{\mathrm{a}}$ & $(11.5 \%)$ \\
Tb.Sp $(\mathrm{mm})$ & $0.25 \pm 0.01^{\mathrm{a}}$ & $(-10.2 \%)$ & $0.25 \pm 0.01$ & $(-8.9 \%)$ & $0.25 \pm 0.01$ & $(-9.3 \%)$ & $0.26 \pm 0.01$ & $(-5.8 \%)$ \\
\hline
\end{tabular}

TS, tail-suspension; TS-RH, tail-suspension with high-dose raloxifene; TS-RM, tail-suspension with medium-dose raloxifene; TS-RL, tail-suspension with low-dose raloxifene; TS-A, tail-suspension with alendronate; BV/TV, bone volume density; Conn.D., connectivity density; SMI, structure model index; Tb.N, trabecular number; Tb.Th, trabecular thickness; Tb.Sp, trabecular plate separation. ${ }^{\mathrm{a}} \mathrm{P}<0.05$, ${ }^{\mathrm{b}} \mathrm{P}<0.01 \mathrm{vs}$. TS group.

A

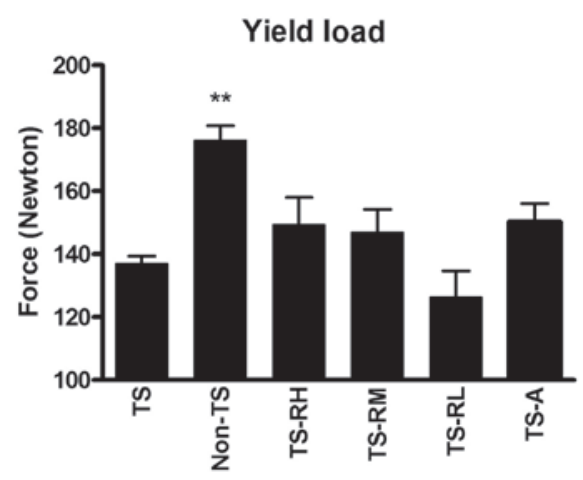

C

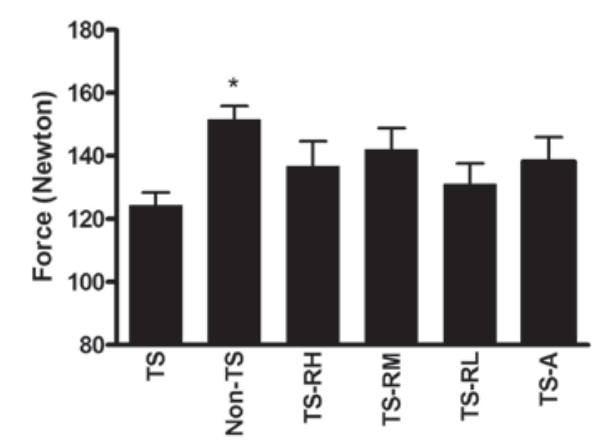

B

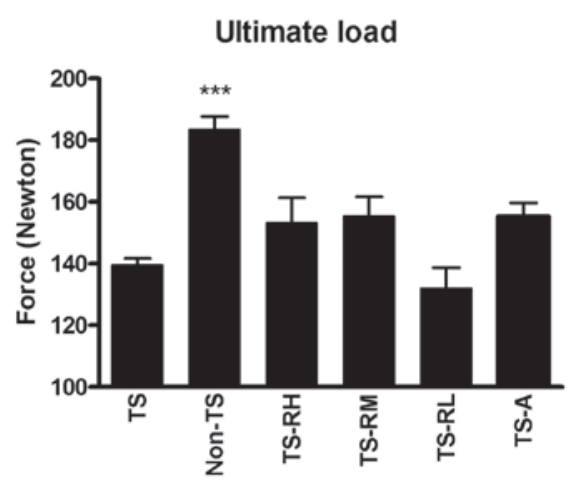

D

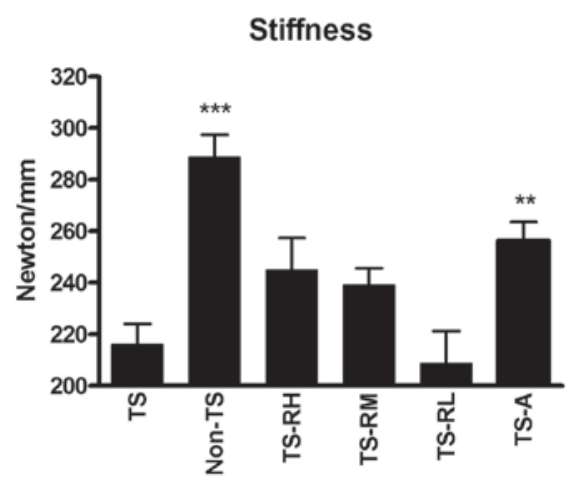

Figure 3. Biomechanical properties of femoral diaphyses of rats from three-point bending test. Mean values are shown and the error bars represent the standard error of mean. (A) Yield load. (B) Ultimate load. (C) Failure load. (D) Stiffness. TS, tail-suspension; Non-TS, non-tail suspension; TS-RH, tail-suspension with high-dose raloxifene; TS-RM, tail-suspension with medium-dose raloxifene; TS-RL, tail-suspension with low-dose raloxifene; TS-A, tail-suspension with alendronate. ${ }^{*} \mathrm{P}<0.05,{ }^{* *} \mathrm{P}<0.01,{ }^{* * * *} \mathrm{P}<0.001$, (vs. TS group).

Proximal tibia. Total tibial BMD was reduced significantly in the TS group ( $\mathrm{P}<0.01$ for both time points), but not in the TS-RM and TS-A groups, throughout the experimental period (Fig. 2A). No significant decrease was observed in the TS-RH group at day 28 and in the TS-RL group at day 14. At day 28, the mean percentage difference of the TS-RH and TS-RM groups was lower than that of the TS group $(\mathrm{P}<0.05$ and $\mathrm{P}<0.001$, respectively).

For the trabecular region, no significant BMD loss was observed in TS-RH, TS-RM and TS-A groups throughout the tail-suspension period (Fig. 2B). The mean percentage difference of the TS-RM group was significantly lower than the TS group at day 28. No significant change of tibial cortical BMD from baseline was found throughout the study in all the drug-treated groups (Fig. 2C).

Similar to the distal femur, no significant difference was observed in BMD in the three regions when raloxifene-treated groups were compared with the TS-A group horizontally in the proximal tibia.

Differences in micro-architecture. Loss of BV/TV and Tb.Th was mitigated significantly when the tail-suspended rats 
were treated with high- or medium-dose raloxifene. These two parameters in the TS-RH and TS-RM groups were significantly higher than those of the TS groups (Table III). The reduction of Tb.N was also retarded when raloxifene was administered. Tb.N was significantly higher in the TS-RM and TS-RL groups than that in the TS group (Table III). Tb.Sp was found to be similar to normal levels $(0.25 \mathrm{~mm}$ as in the Non-TS group, Table II) with raloxifene administration. A significant difference of Tb.Sp was found when the TS-RH group was compared with the TS group $(\mathrm{p}<0.05)$ (Table III). The differences in Tb.Sp between the TS-RM and TS groups, as well as the $\mathrm{S}-\mathrm{RL}$ and TS groups, were marginally significant $(\mathrm{p}=0.097$ and 0.083 , respectively).

In the TS-A group, significant differences in BV/TV, SMI and Tb.Th were observed when compared with the TS group (Table III). Nonetheless, there was no statistical difference in any of the micro-architectural parameters when the TS-A group was compared with the three raloxifene-treated groups.

Biomechanical properties of bone assessed by the three-point bending test. Losses of biomechanical properties were observed in the femoral diaphysis of the TS group. Yield load, ultimate load and failure load in the Non-TS group were significantly higher than those in the TS group (Fig. 3A-C). Raloxifene treatment at high- and medium-dose appeared to retard the loss of these biomechanical properties; however, this was not statistically significant. Stiffness of the femur was also reduced significantly after tail-suspension $(-24.8 \%$ when the TS group was compared with the Non-TS group, $\mathrm{P}<0.001$ ) (Fig. 3D). However, treatment with alendronate prevented the reduction of stiffness and a significant $18.1 \%$ higher stiffness was observed when the TS-A group was compared with the TS group $(\mathrm{P}<0.01)$. Similarly, raloxifene at high- and medium-doses showed, although insignificantly, higher stiffness values than those found in the TS group. There was no significant difference in the four biomechanical parameters when the raloxifene-treated groups were compared with TS-A group.

\section{Discussion}

In order to confirm that the TS rat is a valid animal model for the study of osteoporosis and is comparable to the OVX rat model, a group of OVX rats was also included in this study. The bone material, micro-architectural and biomechanical properties were compared. The changes of BMD in the OVX and TS groups were found to be comparable. The percentage loss of total or trabecular BMD in the femur and tibia of the TS group was similar to that of the OVX group in the 28-day experiment. The region where the highest reduction of BMD occurred was the trabecular region. Higher percentage bone loss was observed in the trabecular region than in the cortical region in the femur and tibia of the two groups. Similar findings were observed by Bloomfield et al (14). The authors reported that cancellous $\mathrm{BMD}$ at the proximal tibia and femoral neck of their rats was significantly lowered by 21 and $20 \%$, respectively, after 28 days of hindlimb unloading. Our results were also consistent with the observation that cosmonauts experience selective loss of cancellous bone mineral density after 6 months of spaceflight (8). In the micro-architectural analysis, almost all parameters of the two groups were similar at the end of the study. This observation was similar to those obtained in a reported study, in which a micro-CT was used to evaluate the bone architecture of immobilized osteoporotic rats (22). From the biomechanical point of view, the bone strengths, including yield, ultimate and failure load of the TS and OVX groups were lower than those of the other groups. These findings revealed that, from the bone material, micro-architectural and biomechanical perspectives, the effects of weightlessness on weight-bearing bones are comparable to those of ovariectomy in experimental rats. This new model is particularly suitable for the study of bone changes in male animals, although orchidectomy has been used as a valid model in previous studies $(23,24)$.

The most frequently tested drugs in pharmaceutical studies using TS rats are bisphosphonates. An $80 \%$ decrease in bone mass was fully prevented by high-dose tiludronate after 23 days of treatment in hindlimb unloaded rats (25). However, concerns have been raised about potential over-suppression of bone turnover during the long-term use of bisphosphonates. There is increasing evidence showing that subtrochanteric or proximal diaphyseal femoral fracture is related to long-term bisphosphonate use $(26,27)$. Jaw osteonecrosis has also been found to be associated with bisphosphonate therapy in recent years $(28,29)$. Risedronate was also reported as being unable to overcome the intense stimulus for osteoclast recruitment in long-term disuse osteoporosis (30).

The effect of raloxifene on disuse osteoporosis has been demonstrated in the present study. Reduction of BMD after tail-suspension in the raloxifene-treated groups was lower than that in the TS group. Raloxifene at $1.25 \mathrm{mg} / \mathrm{kg} /$ day (TS-RM) was the most effective among the three doses tested. It significantly protected the femur and tibia of the rats against bone deterioration within 28 days of tail-suspension. The protection was found not only in the trabecular bone, but also in the cortical bone at the metaphysis. Results showed that there was no significant reduction in total, trabecular and cortical BMD in both the distal femur and proximal tibia of the TS-RM group. The micro-architectural properties of the trabecular bone on the distal femur were also improved by raloxifene at this dosage. Bone volume density, trabecular number and trabecular thickness in the TS-RM group were significantly higher than those in the TS group. These results indicated that raloxifene at $1.25 \mathrm{mg} /$ $\mathrm{kg} /$ day protected the trabeculae against degradation in an environment lacking in biomechanical stimulation. Improvements in material (BMD) and micro-architectural properties of bone by raloxifene resulted in a better bone quality, which was revealed by the biomechanical test. The TS-RM group sustained a higher yield, ultimate and fracture load than the TS group, although such differences were not statistically significant. Raloxifene at $2.5 \mathrm{mg} / \mathrm{kg} /$ day (TS-RH) also showed a preventive effect against bone loss. There was no significant BMD reduction observed at the whole proximal tibia and at the total and cortical regions of the femur in this group, although a significant reduction of trabecular BMD was observed at the distal femur.

These results suggested that raloxifene, although it is a SERM, showed an osteoprotective effect in male animals with disuse osteoporosis. Its pharmaceutical effects on the prevention of bone loss in the state of weightlessness were comparable to those of alendronate. However, the molecular mechanism by which alendronate tackles osteoporosis development is different 
from that of raloxifene $(31,32)$. This aspect should be confirmed by histomorphometry or gene expression in future studies.

The Food and Drug Administration (FDA) has approved three SERMs for clinical use thus far, and only raloxifene has been approved for the prevention and treatment of postmenopausal osteoporosis (33). However, there remains a lack of studies revealing the efficacy of raloxifene on disuse osteoporosis. Using this tail-suspension rat model, the in-depth in vivo mechanisms by which raloxifene tackle disuse osteoporosis are due to be further investigated by means of, for instance, analyses of serum bone markers, histomorphometry, immunohistochemistry and observation of gene expression.

To the best of our knowledge, the present study has for the first time, demonstrated the preventive effects of raloxifene against bone loss in disuse osteoporosis using the tail-suspension rat model. With the administration of raloxifene, deterioration of both material and micro-architectural properties of unloaded trabecular bone were reduced significantly. The reduction of bone strength, however, is alleviated out after 28 days of treatment of disuse osteoporosis. This new animal model, suitable for male and female animals, could be conveniently used in future studies of therapeutic agents against osteoporosis.

\section{Acknowledgements}

The authors would like to acknowledge Ming Lai Foundation and The International Association of Lions Clubs District 303 - Hong Kong and Macau Tam Wah Ching Chinese Medicine Resource Centre for their support to our Institute.

\section{References}

1. Takata S and Yasui N: Disuse osteoporosis. J Med Invest 48 : $147-156,2001$

2. Karlsson MK, Nordqvist A and Karlsson C: Physical activity increases bone mass during growth. Food Nutr Res 52: 2008. doi: 10.3402/fnr.v52i0.1871.

3. Wilsgaard T, Emaus N, Ahmed LA, Grimnes G, Joakimsen RM, Omsland TK and Berntsen GR: Lifestyle impact on lifetime bone loss in women and men: the Tromso Study. Am J Epidemiol 169: 877-886, 2009.

4. Ehrlich PJ and Lanyon LE: Mechanical strain and bone cell function: a review. Osteoporos Int 13: 688-700, 2002.

5. Bikle DD, Sakata T and Halloran BP: The impact of skeletal unloading on bone formation. Gravit Space Biol Bull 6: 45-54, 2003.

6. Tilton FE, Degioanni JJ and Schneider VS: Long-term follow-up of Skylab bone demineralization. Aviat Space Environ Med 51: 1209-1213, 1980.

7. Smith SM, Wastney ME, O'Brien KO, Morukov BV, Larina IM, Abrams SA, Davis-Street JE, Oganov V and Shackelford LC: Bone markers, calcium metabolism, and calcium kinetics during extended-duration space flight on the mir space station. J Bone Miner Res 20: 208-218, 2005.

8. Vico L, Collet P, Guignandon A, Lafage-Proust MH, Thomas T, Rehaillia $\mathrm{M}$ and Alexandre C: Effects of long-term microgravity exposure on cancellous and cortical weight-bearing bones of cosmonauts. Lancet 355: 1607-1611, 2000.

9. Sibonga JD, Evans HJ, Sung HG, Spector ER, Lang TF, Oganov VS, Bakulin AV, Shackelford LC and LeBlanc AD Recovery of spaceflight-induced bone loss: bone mineral density after long-duration missions as fitted with an exponential function. Bone 41: 973-978, 2007.

10. Michaëlsson K, Bergström R, Mallmin H, Holmberg L, Wolk A and Ljunghall S: Screening for osteopenia and osteoporosis: selection by body composition. Osteoporos Int 6: 120-126, 1996

11. Nayak S, Roberts MS and Greenspan SL: Factors associated with diagnosis and treatment of osteoporosis in older adults. Osteoporos Int 20: 1963-1967, 2009.
12. Nelson RE, Nebeker JR, Sauer BC and LaFleur J: Factors associated with screening or treatment initiation among male United States veterans at risk for osteoporosis fracture. Bone 50: 983-988, 2012

13. Morey-Holton ER and Globus RK: Hindlimb unloading of growing rats: a model for predicting skeletal changes during space flight. Bone 22: S83-S88, 1998.

14. Bloomfield SA, Allen MR, Hogan HA and Delp MD: Site- and compartment-specific changes in bone with hindlimb unloading in mature adult rats. Bone 31: 149-157, 2002.

15. Shimano MM and Volpon JB: Biomechanics and structural adaptations of the rat femur after hindlimb suspension and treadmill running. Braz J Med Biol Res 42: 330-338, 2009.

16. Saxon LK, Robling AG, Castillo AB, Mohan S and Turner $\mathrm{CH}$ : The skeletal responsiveness to mechanical loading is enhanced in mice with a null mutation in estrogen receptor-beta. Am J Physiol Endocrinol Metab 293: E484-E491, 2007.

17. Lanyon LE: Using functional loading to influence bone mass and architecture: objectives, mechanisms, and relationship with estrogen of the mechanically adaptive process in bone. Bone 18: 37S-43S, 1996.

18. Ehrlich PJ, Noble BS, Jessop HL, Stevens HY, Mosley JR and Lanyon LE: The effect of in vivo mechanical loading on estrogen receptor alpha expression in rat ulnar osteocytes. J Bone Miner Res 17: 1646-1655, 2002.

19. Licata AA, Ciaccia AV, Wong M and Draper MW: Raloxifene: a new choice for treating and preventing osteoporosis. Cleve Clin J Med 67: 273-280, 2000.

20. Morii H, Ohashi Y, Taketani Y, Fukunaga M, Nakamura T, Itabashi A, Sarkar S and Harper K: Effect of raloxifene on bone mineral density and biochemical markers of bone turnover in Japanese postmenopausal women with osteoporosis: results from a randomized placebo-controlled trial. Osteoporos Int 14: 793-800, 2003.

21. Morey-Holton ER and Globus RK: Hindlimb unloading rodent model: technical aspects. J Appl Physiol 92: 1367-1377, 2002.

22. Laib A, Barou O, Vico L, Lafage-Proust MH, Alexandre C and Rügsegger P: 3D micro-computed tomography of trabecular and cortical bone architecture with application to a rat model of immobilisation osteoporosis. Med Biol Eng Comput 38: 326-332, 2000.

23. Fitts JM, Klein RM and Powers CA: Comparison of tamoxifen and testosterone propionate in male rats: differential prevention of orchidectomy effects on sex organs, bone mass, growth, and the growth hormone-IGF-I axis. J Androl 25: 523-534, 2004.

24. Urasopon N, Hamada Y, Asaoka K, Cherdshewasart W and Malaivijitnond S: Pueraria mirifica, a phytoestrogen-rich herb, prevents bone loss in orchidectomized rats. Maturitas 56: 322-331, 2007.

25. Barou O, Lafage-Proust MH, Martel C, Thomas T, Tirode F, Laroche N, Barbier A, Alexandre C and Vico L: Bisphosphonate effects in rat unloaded hindlimb bone loss model: three-dimensional microcomputed tomographic, histomorphometric, and densitometric analyses. J Pharmacol Exp Ther 291: 321-328, 1999.

26. Neviaser AS, Lane JM, Lenart BA, Edobor-Osula F and Lorich DG: Low-energy femoral shaft fractures associated with alendronate use. J Orthop Trauma 22: 346-350, 2008.

27. Abrahamsen B, Eiken P and Eastell R: Subtrochanteric and diaphyseal femur fractures in patients treated with alendronate: A register-based national cohort study. J Bone Miner Res 24: 1095-1102, 2009.

28. Migliorati CA, Siegel MA and Elting LS: Bisphosphonateassociated osteonecrosis: a long-term complication of bisphosphonate treatment. Lancet Oncol 7: 508-514, 2006.

29. Vieillard MH, Maes JM, Penel G, Facon T, Magro L, Bonneterre J and Cortet B: Thirteen cases of jaw osteonecrosis in patients on bisphosphonate therapy. Joint Bone Spine 75: 34-40, 2008.

30. Li CY, Price C, Delisser K, Nasser P, Laudier D, Clement M, Jepsen KJ and Schaffler MB: Long-term disuse osteoporosis seems less sensitive to bisphosphonate treatment than other osteoporosis. J Bone Miner Res 20: 117-124, 2005.

31. Heringa M: Review on raloxifene: profile of a selective estrogen receptor modulator. Int J Clin Pharmacol Ther 41: 331-345, 2003.

32. Russell RG, Watts NB, Ebetino FH and Rogers MJ: Mechanisms of action of bisphosphonates: similarities and differences and their potential influence on clinical efficacy. Osteoporos Int 19: 733-759, 2008.

33. Riggs BL and Hartmann LC: Selective estrogen-receptor modulators - mechanisms of action and application to clinical practice. N Engl J Med 348: 618-629, 2003. 